\title{
A note on decoding of superimposed codes
}

\author{
Tayuan Huang* Chih-wen Weng ${ }^{\dagger}$
}

September 25, 2003

\begin{abstract}
A superimposed code with general distance $D$ can be used to construct a non-adaptive pooling design. It can then be used to identify a few unknown positives from a large set of items by associating naturally an outcome vector $u$. A simple method for decoding the outcome vector $u$ is given whenever there are at most $\left\lfloor\frac{D-1}{2}\right\rfloor$ errors occuring in the outcome vector $u$. Moreover, another simple method of detecting whether there is any error occuring in the outcome vector $u$ is also given whenever there are at most $D-1$ errors in $u$. Our method is a generalization of the classical result of W. H. Kautz and R. C. Singleton[Nonadaptive binary superimposed codes, IEEE Trans. Inform. Theory, 10:363-377, 1964].
\end{abstract}

Keywords: superimposed codes.

\section{Introduction}

The notion of superimposed codes was first introduced by W. H. Kautz and R. C. Singleton [6] with distance 1 in 1964, and then by A. G. D'yachkov, V. V. Rykov and A. M. Rashad for general distance [4] around 1989. In addition to some applications found in [1], superimposed codes have become a dominating tool in a recent study of non-adaptive group testings, and have attracted more attentions nowadays due to its recent application to

*Department of Applied Mathematics, National Chiao Tung University, Taiwan R.O.C.

${ }^{\dagger}$ Department of Applied Mathematics, National Chiao Tung University, Taiwan R.O.C. 
pooling designs in DNA mapping, see [2] for more details. A uniform way of constructing a class of superimposed codes with distance 1 was given by A. J. Macula [8] in 1996. Two families of superimposed codes with general distance were found by H. Ngo and D. Du [7]. It was soon generalized over a class of ranked posets, called pooling spaces, by the authors [5] to find the superimposed codes with general distance.

A superimposed code with general distance $D$ can be used to construct a non-adaptive pooling design. It can then be used to identify a few positive items from a large set of items by associating naturally an outcome vector $u$. The purpose of this article is to give a simple method for decoding the outcome vector $u$ to identify those positives correctly whenever there are at most $\left\lfloor\frac{D-1}{2}\right\rfloor$ errors occurring in the outcome vector $u$. Moreover, another simple method of detecting whether there is any error occurring in the outcome vector $u$ is also given whenever there are at most $D-1$ errors in $u$. Our method is a generalization of the classical result of W. H. Kautz and R. C. Singleton [6].

\section{Preliminaries}

For a positive integer $m$, set $[m]:=\{1,2, \cdots, m\}$. Fix four positive integers $t, n, D, d$ with $D \leq t$ and $d \leq n$. A superimposed code $M$ with length $t$, volume $n$, distance $D$ and strength $d$ is a family $M=\left\{C_{1}, C_{2}, \cdots, C_{n}\right\}$ of $n$ subsets of $[t]$ such that for any index subset $S \subseteq[n]$ with $|S| \leq d$ and any $i \in[n] \backslash S$,

$$
\left|C_{i}-\bigcup_{j \in S} C_{j}\right| \geq D
$$

The $t \times n$ incidence matrix of a superimposed code $M$ with length $t$, volume $n$, distance $D$ and strength $d$ is called the $(d, e)$-disjunct matrix (or $d^{e}$-disjunct matrix ) of size $t \times n$ where $e=D-1$, and if $D=1$ it is called a $d$-disjunct matrix [3], [5].

Throughout the note $M=\left\{C_{1}, C_{2}, \cdots, C_{n}\right\}$ is a superimposed code with length $t$, volume $n$, distance $D$ and strength $d$. $M$ can be used to construct a non-adaptive group testing design on $n$ items by associating the set $[n]$ with 
the set of items and the set $[t]$ with the set of tests. If $i \in C_{j}$ then item $j$ is contained in test $i$. By a set of positives we mean a subset $S \subseteq[n]$ such that $|S| \leq d$. Let $S$ be a set of positives. The ideal output o(S) of $S$ in $M$ is defined by

$$
o(S):=\bigcup_{j \in S} C_{j}
$$

and the test result (or outcome vector) $u$ of $S$ under $M$ is any subset of $[t]$. The number of test errors in the test result $u$ of $S$ under $M$ is the Hamming distance $\partial(u, o(S))$, where

$$
\partial(u, o(S)):=|u-o(S)|+|o(S)-u| .
$$

Suppose the test result $u$ of $S$ under $M$ does not contain any error, or equivalently $u=o(S)$. W. H. Kautz and R. C. Singleton showed the set $S$ of positives can be determined by the test result $u$ [6]. In next section we will generalize their result to allow the test result $u$ containing a few errors.

\section{The Decoding Method}

The methods in decoding and in error detecting of a test result are given in this section. We need a lemma first.

Lemma 3.1. Let $M=\left\{C_{1}, C_{2}, \cdots, C_{n}\right\}$ denote a superimposed code with length $t$, volume $n$, distance $D$ and strength $d$. Let $S, T \subseteq[n]$ be two distinct subsets with each at most d elements. Then the Hamming distance of the ideal outputs $o(S), o(T)$ of $S, T$ respectively under $M$ is at least $D$.

Proof. At least one of $S-T, T-S$ is nonempty, so assume $S-T \neq \emptyset$. Pick $i \in S-T$. By construction

$$
\left|C_{i}-\bigcup_{j \in T} C_{j}\right| \geq D
$$

Referring to notation in $(2.2)$, we find $\partial(o(S), o(T)) \geq D$. This proves the lemma.

The following theorem is the main idea. 
Theorem 3.2. Let $M=\left\{C_{1}, C_{2}, \cdots, C_{n}\right\}$ denote a superimposed code with length $t$, volume $n$, distance $D$ and strength $d$. Suppose $S \subseteq[n]$ with $|S| \leq d$ and $u \subseteq[t]$. Set

$$
T=\left\{j|| C_{j}-u \mid \leq\left\lfloor\frac{D-1}{2}\right\rfloor\right\} .
$$

Then the following (i)-(ii) hold.

(i) Suppose $\partial(o(S), u) \leq\left\lfloor\frac{D-1}{2}\right\rfloor$. Then $T=S$.

(ii) Suppose $\partial(o(S), u) \leq D-1$ and $|T| \leq d$. Then $o(S)=u$ if and only if $o(T)=u$.

Proof. (i) () Pick $j \in S$. Then $C_{j} \subseteq o(S)$ by (2.2). Hence

$$
\begin{aligned}
\left|C_{j}-u\right| & \leq|o(S)-u| \\
& \leq \partial(o(S), u) \\
& \leq\left\lfloor\frac{D-1}{2}\right\rfloor .
\end{aligned}
$$

Thus $j \in T$ by (3.1).

$(\subseteq)$ Pick $j \in T$. Suppose $j \notin S$. By the construction of $M$, there are at least $D$ elements in $C_{j}-o(S)$. Since $\partial(o(S), u) \leq\left\lfloor\frac{D-1}{2}\right\rfloor$, there are at least

$$
D-\left\lfloor\frac{D-1}{2}\right\rfloor=\left\lfloor\frac{D-1}{2}\right\rfloor+1
$$

elements in $C_{j}-u$, a contradiction to (3.1).

(ii) This is clear if $S=T$. Suppose $S \neq T$. Then $\partial(o(S), u)>\left\lfloor\frac{D-1}{2}\right\rfloor$ by (i). In particular, $o(S) \neq u$. Applying triangular inequality and using Lemma 3.1 we find

$$
\begin{aligned}
\partial(o(T), u) & \geq \partial(o(T), o(S))-\partial(o(S), u) \\
& \geq D-(D-1) \\
& =1 .
\end{aligned}
$$

Hence $o(T) \neq u$.

Remark 3.3. The special case $D=1$ in Theorem 3.2 is Kautz and Singleton's result in $1964[6]$. 


\section{A decoding algorithm:}

Suppose $[n]$ is the set of items and $S \subseteq[n]$ with $|S| \leq d$ is the set of positives to be identified. A superimposed code $M$ with length $t$, volume $n$, distance $D$ and strength $d$ for some positive integers $t, D$ is on hand. Let $u$ be the test result of $S$ under $M$, and $o(S)$ be the unknown ideal output of $S$. The Hamming distance $\partial(u, o(S))$ is simplify the number of test errors occuring in the testing procedure. Then do the following:

(i) Determine $T$ first by (3.1) and then determine $o(T)$ by (2.2);

(ii) Suppose there are at most $\left\lfloor\frac{D-1}{2}\right\rfloor$ errors in the outcome vector $u$. Then $T=S$ is concluded by applying Theorem 3.2(i);

(iii) Check where there is an error in the outcome vector $u$ by applying Theorem 3.2(ii) whenever there are at most $D-1$ errors in $u$;

(iv) If $|T|>d$, there is an error; otherwise an error occurs in $u$ if and only if $o(T) \neq u$.

\section{ACKNOWLEDGEMENT}

The authors thank an anonymous referee of the preprint [5] for enlightening the line of study and many valuable ideas.

\section{References}

[1] L. A. Bassalygo, M. S. Pinsker. Limited multiple-access of a nonsynchronous channel. Promlemy Peredachi Informatsii, 19, 8:92-96, 1983(in Russian).

[2] Ding-Zhu Du, Frank K. Hwang. Combinatorial group testing and its applications. Series on applied mathematics v. 12. World Scientific, River Edge, NJ, 2000.

[3] A. G. D'yachkov, A.J. Macula and P. A. Vilenkin. Nonadaptive Group Testing with Error-Correction $d^{e}$-Disjunct Inclusion Matrices. preprint.

[4] A. G. D'yachkov, V.V. Rykov and A. M. Rashad. Superimposed Distance Codes. Prob. of Control and Inform. Theory, 18:237-250, 1989. 
[5] T. Huang and C. Weng. Pooling Spaces and Non-Adaptive Pooling Designs. Submitted to Discrete Math.

[6] W. H. Kautz and R. C. Singleton. Nonadaptive binary superimposed codes. IEEE Trans. Inform. Theory, 10:363-377, 1964.

[7] H. Ngo and D. Du. New Constructions of Non-Adaptive and ErrorTolerance Pooling Designs. Discrete Math., 243:161-170, 2002.

[8] A. J. Macula. A simple construction of $d$-disjunct matrices with certain constant weights. Discrete Math., 162:311-312, 1996. 